\title{
Real-Time MR Diffusion Tensor and Q-Ball Imaging Using Kalman Filtering
}

\author{
C. Poupon ${ }^{1,2}$, F. Poupon ${ }^{1,2}$, A. Roche ${ }^{1,2}$, Y. Cointepas ${ }^{1,2}$, \\ J. Dubois ${ }^{3}$, and J.-F. Mangin ${ }^{1,2}$ \\ ${ }^{1}$ CEA Neurospin - Bât. 145, 91191 Gif-sur-Yvette, France \\ cyril.poupon@cea.fr \\ ${ }^{2}$ IFR49, 91191 Gif-sur-Yvette, France \\ ${ }^{3}$ Faculté de médecine, Université de Genève, Switzerland
}

\begin{abstract}
Magnetic resonance diffusion imaging (dMRI) has become an established research tool for the investigation of tissue structure and orientation. In this paper, we present a method for real time processing of diffusion tensor and Q-ball imaging. The basic idea is to use Kalman filtering framework to fit either the linear tensor or Q-ball model. Because the Kalman filter is designed to be an incremental algorithm, it naturally enables updating the model estimate after the acquisition of any new diffusionweighted volume. Processing diffusion models and maps during ongoing scans provides a new useful tool for clinicians, especially when it is not possible to predict how long a subject may remain still in the magnet.
\end{abstract}

\section{Introduction}

Magnetic resonance (MR) diffusion imaging has become an established technique for inferring structural anisotropy of tissues and mapping the white matter connectivity of the human brain [1. The term diffusion refers to the Brownian motion of water molecules inside tissues that results from the thermal energy carried by these molecules. MR images can be sensitized to that physiological phenomenon from the application of a specific pair of well-known diffusion gradients together with a spin echo pulse sequence.

Technically, diffusion imaging requires the acquisition of a set of diffusion sensitized images from which molecules displacement probability is inferred. Several mathematical models have been designed, becoming more and more complex over the last decade while attempting to make less and less assumptions. In this paper, we focus on both the diffusion tensor (DTI) model (historically the first) introduced by Basser [2] and the Q-ball model (QBI) introduced by Tuch [3]. Despite the huge amount of assumptions (unrestricted environment, structural homogeneity within voxels), the DTI model is still widely used because it can be used in a clinically acceptable time (a few minutes for an entire brain coverage) and provides useful information to the clinicians about the average translational motion (apparent diffusion coefficient, ADC), the anisotropy of white matter structure (fractional anisotropy index, FA), and the RGB orientation map (RGB)). Today, clinical studies of brain pathologies (either neurodegenerative or psychiatric) involve statistical analysis of ADC and FA maps. Q-ball 
belongs to the class of high angular resolution diffusion imaging (HARDI) models that aim at solving the partial voluming problem due to the existence of several putative populations of fibres within a voxel. Such models have been developed to address the inference of white matter connectivity mapping from the knowledge of local microstructural orientations of tissue.

Compared to DTI, QBI requires from five to ten times more diffusion gradient orientations with a higher b-value and therefore cannot be considered as reliable for clinical use for many reasons. First, clinical protocols generally involve different $\mathrm{MR}$ acquisitions (T1, T2, BOLD) limitating the time alloted to diffusion imaging. Second, the patient may move severely during the acquisition (a frequent situation for patients impaired with Huntington disease, Parkinson disease, schizophrenia), hence increasing the risk of aborting the scanning. The same problem arises for studies involving newborns who cannot be sedated: generally, less than $75 \%$ of the subjects can be exploited because they often wake up inside the magnet, due to the level of noise. The opposite situation is also true: the patient can be more cooperative than hypothesized first and it's worth starting with a high b-value DTI scan and continuing with a QBI scan if the patient is still.

This paper addresses the feasibility of real time DTI and QBI processing for displaying reconstructed associated maps during an ongoing scan. This will make it possible to start the scan estimating both models, to cancel the acquisition at any time, or to sustain the scanning when the subject is still in the magnet. If the scanning is stopped after too few diffusion gradient orientations, none of the model is exploitable. Then, according to the acquired number of orientations, either DTI model, QBI model or both can be obtained. To our knowledge, real time processing was previously addressed for BOLD functional imaging [4], but has never been proposed for diffusion imaging.

DTI and QBI models can be expressed in the light of the general linear model framework (GLM) assuming a white noise model. Among available techniques for solving least-squares linear regression models, the Kalman filter provides an appropriate answer to the real time requirement, as it is an incremental solver. After the acquisition of the entire volume for each diffusion gradient orientation, this filter can update DTI and QBI maps, provide variance of the estimate and can deliver an immediate feedback to the clinician or to the expert in cognitive neurosciences.

After introducing the linear models for DTI and QBI in section 2.1, we describe the Kalman filter-based algorithm implemented in section 2.2. Then we focus on the optimization of the diffusion gradient orientation set in section 2.3. In section 3, we give a setup of the realtime protocol used and we illustrate the technique using DTI and QBI MR data, before concluding.

\section{Methods}

\subsection{Model Fitting Formulation}

Let us consider the vector $\boldsymbol{m}=\left[m_{1}, \ldots, m_{N}\right]$, acquired during the acquisition corresponding to the diffusion-sensitized signal measured with the different 
diffusion gradient orientations at a given voxel in the scanned volume. The gradient orientations $\boldsymbol{o}_{i}$ are indexed by $i$ corresponding to the time rank during the acquisition and are numbered from 1 to $N$. The choice of the orientation set will be discussed later in section 2.3. The magnitude of the sensitization is given by the b-value in $s / \mathrm{mm}^{2}$. We also define $m_{0}$ corresponding to the unweighted signal measured with diffusion gradients off.

\section{Tensor general linear model}

The DT model states that the diffusion of water molecules can be considered as free, yielding a Gaussian probability density function characterized by a second order tensor $\boldsymbol{D}$. The signal attenuation observed when applying a diffusion gradient along the normalized direction $\boldsymbol{o}=\left[o_{x}, o_{y}, o_{z}\right]^{T}$ of the space and with sensitization $b$ is exponential:

$$
m=m_{0} e^{-b \boldsymbol{o}^{T} \boldsymbol{D o}}+\mu
$$

where $\mu$ represent the acquisition noise that usually follows a Rician distribution. Taking the natural logarithm of this attenuation, we easily obtain the general linear model:

$$
\boldsymbol{y}=\boldsymbol{B d}+\boldsymbol{\epsilon}
$$

where we define the measured vector of attenuations $\boldsymbol{y}=\left[y_{1}, \ldots, y_{N}\right]^{T}$, with $y_{i}=\log \left(m_{0} / m_{i}\right)$ and $\boldsymbol{d}=\left[D_{x x}, D_{x y}, D_{x z}, D_{y y}, D_{y z}, D_{z z}\right]^{T}$ being the vector of the six unknown coefficients of the diffusion tensor. $\boldsymbol{B}$ is a $N \times 6$ matrix called the diffusion sensitization matrix, built from $N$ rows $\boldsymbol{b}_{\mathbf{1}}, \ldots, \boldsymbol{b}_{\boldsymbol{N}}$ depending only on the diffusion gradient settings $\boldsymbol{b}_{\boldsymbol{i}}=b_{i}\left[o_{x, i}{ }^{2}, 2 o_{x, i} o_{y, i}, 2 o_{x, i} o_{z, i}, o_{y, i}{ }^{2}, 2 o_{y, i} o_{z, i}, o_{z, i}{ }^{2}\right]$.

$\epsilon$ is the $N \times 1$ vector of errors $\epsilon_{i}=-\ln \left(1+\mu e^{b_{i} \boldsymbol{o}_{i}{ }^{T} \boldsymbol{D} \boldsymbol{o}_{i}} / m_{0}\right) \approx-\mu e^{b_{i} \boldsymbol{o}_{i}{ }^{T} \boldsymbol{D} \boldsymbol{o}_{i}} / m_{0}$. Theoretically, the noise model depends on the unknowns as well as on the Rician noise $\mu$, but we assume it is not far from a Gaussian distribution. Studying the true distribution of the noise must be done, but it is not the purpose of this paper that deals with the real-time aspect of the algorithm, even if the estimate is not statistically optimum.

\section{Q-ball general linear model}

The Q-ball model states that the orientation distribution function (ODF) $\psi(\boldsymbol{o})=$ $\int_{0}^{\infty} p(r \boldsymbol{o}) d r$ that gives the likelihood of any orientation $\boldsymbol{o}$ can be obtained by sampling a sphere in the Q-space [3] which radius is set up by a high b-value (typically greater than $3000 \mathrm{~s} / \mathrm{mm}^{2}$ ) with a huge number of gradient orientations (from 160 to 500 according to the litterature). A good approximation of the ODF was proposed by Tuch using the Funk-Radon transform (FRT). In order to obtain $\psi\left(\boldsymbol{o}_{\boldsymbol{i}}\right)$, the FRT integrates the MR signal along the equator of the given orientation $\boldsymbol{o}_{\boldsymbol{i}}$.

A first linear model of the FRT has been published in [5] corresponding to the raw algorithm. More recently, Descoteaux et al [6] proposed an elegant reformulation of the FRT using the Funk-Hecke theorem for decomposing the ODF onto a symmetric, orthonormal and real spherical harmonics. 
Let $\boldsymbol{c}=\left[c_{1}, \ldots, c_{K}\right]^{T}$ be the $K \times 1$ vector of coefficients $c_{j}$ of the spherical harmonics decomposition of the ODF and is calculated from the reconstruction equation:

$$
\boldsymbol{c}=\boldsymbol{P}\left(\boldsymbol{B}^{T} \boldsymbol{B}+\lambda \boldsymbol{L}\right)^{-1} \boldsymbol{B}^{T} \boldsymbol{m}
$$

where $\boldsymbol{B}$ is a $N \times K$ matrix built from the modified spherical harmonics basis $B_{i j}=Y_{j}\left(\theta\left(\boldsymbol{o}_{\boldsymbol{i}}\right), \phi\left(\boldsymbol{o}_{\boldsymbol{i}}\right)\right)(\theta$ is the colatitude and $\phi$ is the azimuth of the diffusion gradient orientation $\left.\boldsymbol{o}_{\boldsymbol{i}}\right), \boldsymbol{L}$ is the $K \times K$ matrix of Laplace-Beltrami regularization operator, $\lambda$ is the regularization factor, $\boldsymbol{P}$ is the $K \times K$ Funk-Hecke diagonal matrix with elements $P_{i i}=2 \pi P_{l(j)}(0) / P_{l(j)}(1)\left(P_{l(j)}(x)\right.$ is the Legendre polynomial of degree $l(j)$, see also [6] for the definition of $l(j)))$.

From the knowledge of the decomposition $\boldsymbol{c}$, we can obtain the ODF value for the orientation $\boldsymbol{o}$ calculating the composition (4):

$$
\boldsymbol{\psi}(\boldsymbol{o})=\sum_{j=1}^{N} c_{j} Y_{j}(\theta(\boldsymbol{o}), \phi(\boldsymbol{o}))
$$

The equation (3) can easily be reversed to get the general linear model:

$$
\boldsymbol{m}=\boldsymbol{B}^{+} \boldsymbol{c}+\boldsymbol{\epsilon} \quad \text { with } \quad \boldsymbol{B}^{+}=\left(\boldsymbol{P}\left(\boldsymbol{B}^{T} \boldsymbol{B}+\lambda \boldsymbol{L}\right)^{-1} \boldsymbol{B}^{T}\right)^{\dagger}
$$

where $\epsilon$ is the vector of Rician acquisition noise that we assume to be Gaussian in order to stay in the ordinary linear least square framework. The ()$^{\dagger}$ stands for the Moore-Penrose pseudo-inverse operator. Further investigation must be done concerning this operator in order to prevent the apparition of negative items in the vector $c$ when the spherical harmonics order is increased.

\subsection{Kalman Filtering}

The Kalman filter is a recursive solver that optimally minimize the mean square error of the estimation [7] 8. Because of its recursive nature, it is a suitable method for updating the DTI or QBI model parameters after the acquisition of each new diffusion-sensitized volume. Moreover, the Kalman filter provides, at each time frame, an estimated covariance of the parameter estimate that can be used to automatically stop the ongoing scan when the maximum variance falls below a minimum threshold.

In section 2.1, we obtained two general linear models for DTI and QBI of the form $\boldsymbol{y}=\boldsymbol{A} \boldsymbol{x}+\boldsymbol{\epsilon}$. The Kalman filter exploits any new measure $y$ for updating the unknown parameters $\boldsymbol{x}$, usually called the state vector.

Assume that after the acquisition of rank $i$, a current estimate $\hat{\boldsymbol{x}}(i-1)$ is available. Given the new MR measurement $y(i)$ and the vector $\boldsymbol{a}(i)=\left[A_{i 1}, \ldots, A_{i P}\right]^{T}$ corresponding to the $i^{\text {th }}$ row of the matrix $\boldsymbol{A}$, the innovation $\nu(i)=y(i)-$ $\boldsymbol{a}(i)^{T} \hat{\boldsymbol{x}}(i-1)$ is calculated. The Kalman filter then updates the parameters using the recursion:

$$
\left\{\begin{array}{l}
\boldsymbol{k}(i)=\left(1+\boldsymbol{a}(i)^{T} \boldsymbol{P}(i-1) \boldsymbol{a}(i)\right)^{-1} \boldsymbol{P}(i-1) \boldsymbol{a}(i) \\
\hat{\boldsymbol{x}}(i)=\hat{\boldsymbol{x}}(i-1)+\nu(i) \boldsymbol{k}(i) \\
\boldsymbol{P}(i)=\boldsymbol{P}(i-1)-\boldsymbol{k}(i) \boldsymbol{a}(i)^{T} \boldsymbol{P}(i-1)
\end{array}\right.
$$


where the vector $\boldsymbol{k}(i)$ is usually called the Kalman gain. $\boldsymbol{P}(i)$ represents an estimate of the normalized covariance matrix of $\boldsymbol{x}$ given the information at time i. The unnormalized covariance of $\hat{\boldsymbol{x}}(i)$ is equal to $\hat{\sigma}(i)^{2} \boldsymbol{P}(i)$ using the recursion:

$$
\hat{\boldsymbol{\sigma}}(i)=\frac{i-1}{i}\left[\hat{\boldsymbol{\sigma}}(i-1)+\nu(i)^{2}\left(1+\boldsymbol{a}(i)^{T} \boldsymbol{P}(i-1) \boldsymbol{a}(i)\right)^{-1}\right]
$$

The initial guesses $\hat{\boldsymbol{x}}(0), \boldsymbol{P}(0)$ and $\hat{\sigma}(0)$ can be respectively set to the null vector, the identity matrix and zero.

\subsection{Optimum Diffusion Gradient Orientation Set}

Contrary to functional scans where the time order of the stimuli cannot be modified, diffusion scans can play the diffusion gradient orientation set in random order, provided it is a uniform distribution of the orientations in the tridimensional space, for obtaining an accurate tensor or Q-ball estimation.

The optimum orientation count is still debated in the litterature 9]. Increasing this number directly improves the SNR of the ADC, FA and ODF maps, at the price of a longer scan time and knowing that it is not always possible to predict how long a subject will remain still in the magnet. In order to reduce the risk of failure, we implemented the sequence of orientations proposed in [10, which yields the "best" spatial distribution of the orientations, should the acquisition be terminated before completion. This sequence consists in a series of small meaningful subsets of 14 uniform orientations, while all clusters complement each other with additional orientations. Figure 1 gives an example of 42 orientations divided into 3 subsets of 14 orientations. The distribution obtained from the 14 or 28 orientation subsets are more uniform with the optimum distribution than with the conventional distribution. This strategy has been applied only for DTI scanning, but can be also used for QBI scanning.

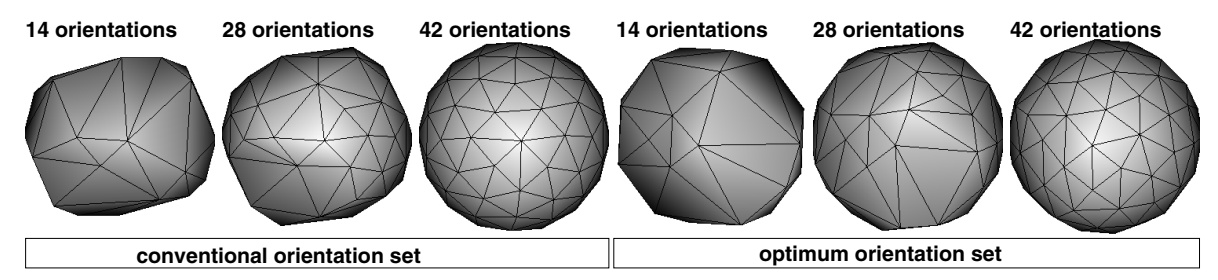

Fig. 1. Comparison of conventional and optimum sets of 42 orientations; meshes of the full distributions are represented as well as meshes corresponding to subsets restrained to the 14 or 28 first orientations; conventional set is more uniform than the optimum set when the full set is acquired, but less uniform when it is not complete; optimum set is to be prefered for real time scan that can be interrupted at any time

\subsection{DTI and QBI Acquisition Settings}

The real time diffusion Kalman filter was evaluated on an adult, under a protocol approved by the Institutional Ethical Committee. Two different acquisitions 
were performed using a DW Dual Spin Echo EPI technique on a 1.5T MRI system (ExciteII, GE Healthcare, USA) for validating both DTI and QBI solvers. Pulse sequence settings were $b=700 \mathrm{~s} / \mathrm{mm} 2,42$ optimum gradient orientation set, matrix $128 \times 128$, 60 slices, $F O V=24 \mathrm{~cm}$, slice thickness $T H=2 \mathrm{~mm}$, $T E / T R=66.2 \mathrm{~ms} / 12.5 \mathrm{~s}$ for a DTI scan time of $9 \min 48 \mathrm{~s}$, and $b=3000 \mathrm{~s} / \mathrm{mm}^{2}$, 200 conventional gradient orientation set, matrix $128 \times 128$, 60 slices, $F O V=$ $24 \mathrm{~cm}$, slice thickness $T H=2 \mathrm{~mm}, T E / T R=93.2 \mathrm{~ms} / 19 \mathrm{~s}$ for a QBI scan time of $72 \min 50 \mathrm{~s}$.

\section{Results}

\subsection{Real Time Standard Diffusion Maps}

At each iteration of the DTI scan, an approximation of the diffusion tensor is available for each voxel of the brain. Therefore it is possible to process its eigensystem online and then to estimate the ADC / FA / RGB maps. Columns 1-3 of figure 2 depict the evolution of these maps during the ongoing scan. For comparison, the $4^{\text {th }}$ column shows the result of a standard offline SVD analysis. There is no qualitative difference with the $3^{\text {rd }}$ column processed using the Kalman filter.
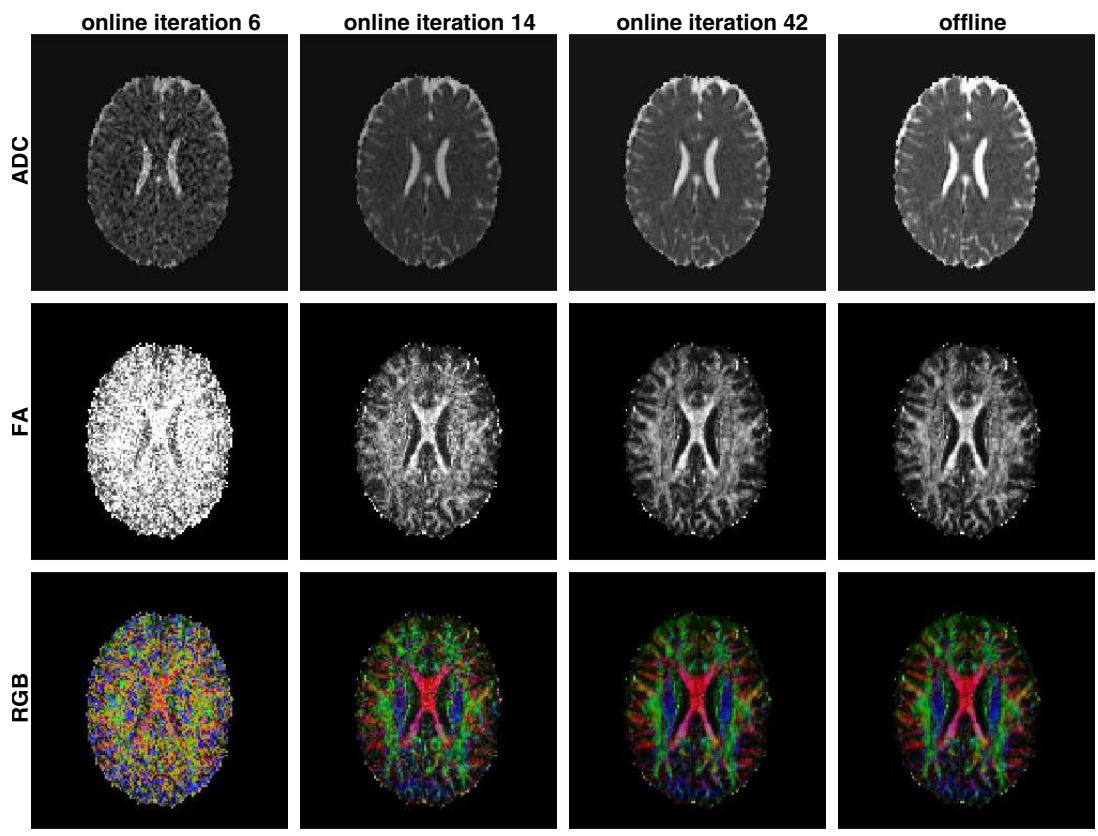

Fig. 2. Real time processing of ADC / FA / RGB maps using the DTI Kalman filter during an ongoing DTI scan at $b=700 \mathrm{~s} / \mathrm{mm}^{2}$ with 42 diffusion gradient orientations; the columns $1 / 2 / 3$ correspond to iteration 6,14 and 42 ; the last column shows the result of the standard offline processing 


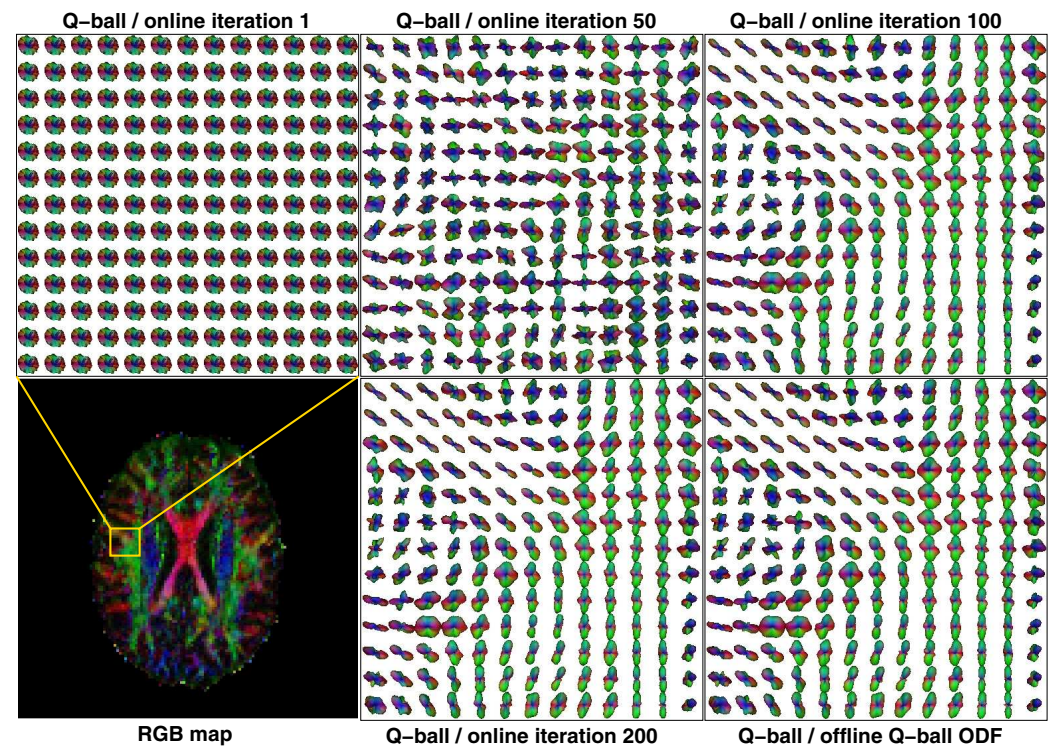

Fig. 3. Real time processing of a Q-ball ODF map using the QBI Kalman filter during an ongoing QBI scan at $b=3000 \mathrm{~s} / \mathrm{mm}^{2}$ with 200 diffusion gradient orientations; the bottom row displays a RGB orientation map (left) on which is drawn a region of interest inside the white matter, containing fibre crossings and homogeneous voxels, and the corresponding map of Q-ball ODFs (right) processed with the offline routine; the top row shows iterations 1, 50 and 200 of the same ODF map calculated with the online Kalman filter

The use of an optimum orientation set speeds up the convergence of the estimation that can be considered exploitable by clinicians from the $14^{\text {th }}$ iteration. The time required to perform one iteration of the DTI Kalman filter over the full brain is less than 8 seconds on a $3.2 \mathrm{GHz}$ linux station, which is lower than the repetition time $T R=12.5 \mathrm{~s}$ of the scan. Consequently, there is no addtional delay between two consecutive acquisitions, making this protocol truly real time.

\subsection{Real Time Orientation Distribution Function Maps}

The Q-ball online Kalman filter was used for processing ODFs during the ongoing QBI scan. A symmetrical spherical harmonics basis of order 8 was chosen and the Laplace-Beltrami regularization factor was set to 0.006 as proposed in [6]. The ODFs are reconstructed on 400 normalized uniform orientations. The QBI dedicated Kalman filter (5) provides, at each step and for each voxel of the brain, an estimate of the decomposition of the ODF on to a symmetric spherical harmonics basis from which it is easy to obtain the values for any orientation $\boldsymbol{o}$ of the space (equation (4)).

The top row on figure 3 shows the evolution of the ODF map during the Kalman recursion on a region of interest contained in the subcortical white 
matter, and exhibiting some fibre crossings as well as voxels with homogeneous fibre populations. As for DTI, there is no qualitive difference between the ODF maps obtained from the online Kalman filter or from the offline Q-ball algorithm given by equation (3). The choice for that order can be discussed, because there are 45 spherical harmonic coefficients to estimate, which represents a lot of unknown, and consequently requires a lot of iterations before to converge. A lower order would give nice results after less iterations.

The time required for performing one iteration of the QBI Kalman filter on a slice is almost 5 seconds, which is lower than the repetition time $T R=19 \mathrm{~s}$ of the scan. Obviously, it is more time consuming than DTI processing where the entire brain can be processed within 8 seconds. In the case of QBI, only 4 slice locations can be performed in real time. However, the $\mathrm{C}++$ code can still be optimized and parallelized on a grid of processors, if the whole brain is to be processed in real time.

\section{Conclusion}

We have developed an incremental Kalman-filter based framework dedicated to real-time diffusion MR imaging. This framework address both diffusion tensor and Qball models, and enables processing the standard DTI / QBI maps, in real time during an ongoing scan. The methodology developed in this paper is very suitable for clinical use when a quick feedback is required during the acquisition or when the cooperation of the subject is not certain. More quantitative evaluations of the difference between online and offline reconstructions must be performed for validating this approach, as well as studying more deeply the underlying model of noise present in DTI and QBI data, which was not the main purpose of this paper. There is also a clear need to study the best trade-off between the iteration number, the wavevector number 11, the regularization factor, and estimation order. A future extension of this work entails online fibre tracking. To that end, we plan to modify the diffusion Kalman filter in order to process incremental connectivity maps during ongoing diffusion scans.

\section{References}

1. LeBihan, D., Breton, E., Lallemand, D.: MR imaging of intravoxel incoherent motions: application to diffusion and perfusion in neurologic disorders. Radiology 161, 401-407 (1986)

2. Basser, P.J., Mattiello, J., Le Bihan, D.: Estimation of the effective self-diffusion tensor from the NMR spin echo. Journal of Magnetic Resonance 103, 247-254 (1994)

3. Tuch, D.: Diffusion MRI of complex tissue structure. PhD thesis, Harvard-MIT (2002)

4. Roche, A., Pinel, P., Dehaene, S., Poline, J.-B.: Solving incrementally the fitting and detection problems in fMRI time series. In: Barillot, C., Haynor, D.R., Hellier, P. (eds.) MICCAI 2004. LNCS, vol. 3217, pp. 719-726. Springer, Heidelberg (2004)

5. Tuch, D.: Q-ball imaging. Magn. Reson. Med. 52, 1358-1372 (2004) 
6. Descoteaux, M., Angelino, E., Fitzgibbons, S., Deriche, R.: A fast and robust ODF estimation algorithm in Q-ball imaging. In: Proc. ISBI 2006, Arlington, USA, pp. 81-84 (2006)

7. Ayache, N.: Artificial vision for mobile robots. The MIT Press, Cambridge, USA (1991)

8. Welch, G., Bishop, G.: An Introduction to the Kalman Filter. In: SIGGRAPH 2001 course 8, In Computer Graphics, Annual Conference on Computer Graphics \& Interactive Techniques, Cambridge, USA (1991)

9. Jones, D.: The effect of gradient sampling schemes on measures derived from diffusion tensor MRI: a Monte Carlo study. Magn. Reson. Med. 51, 807-815 (2004)

10. Dubois, J., Poupon, C., Lethimonnier, F., Le Bihan, D.: Optimized diffusion gradient orientation schemes for corrupted clinical DTI data sets. MAGMA 19, 134-143 (2006)

11. Khachaturian, M.-H., Wisco, J.-J., Tuch, D.: Boosting the sampling efficiency of q-Ball imaging using multiple wavevector fusion. Magn. Reson. Med. 57, 289-296 (2007) 